\title{
Inhaltsverzeichnis
}

\section{Die Cholera.}

Vorwort . . . . . . . . . . . . . . . . . III-IV

Einleitung. . . . . . . . . . . . . . . . . . . . . . . . . $1-12$

\& 1. Das hippokratische Bild der Cholera . . . . . . . . . . 1-2

§ 2. Die Cholera bei Celsus, Galen, Aretaens . . . . . . . . . 2-5

\& 3. Die Lehre von den Ursachen der Cholera im Altertum und

Mittelalter . . . . . . . . . . . . . . . . . . 5- 6

§ 4. Sporadische und epidemische Cholera . . . . . . . . . . 6- 7

$\S$ 5. Cholera vera, Cholera spuria, Choleralarven . . . . . . . $7-9$

§ 6. Die pandemische Cholera aus Indien . . . . . . . . . . 9-10

$\S$ 7. Das Wort Cholera und sein Inhalt . . . . . . . . . . . 10-12

Erster Teil: Die einheimische Cholera.

I. Hauptstück: Begriff und Geschichte der einheimischen Cholera.

§ 8. Vorkommen der einheimischen Cholera . . . . . . . . . 13-14

§ 9. Ältere Epidemien der Cholera nostras . . . . . . . . . . 14-16

§10. Jüngere Epidemien der Cholera nostras . . . . . . . . . 17-19

II. Hauptstück: Epidemiologie der einheimischen Cholera.

$\S 11$. Zeit und Ort ihrer Ausbrüche . . . . . . . . . . . . 19-21

§12. Die Anlage zur Krankheit . . . . . . . . . . . . . . 21-24

III. Hauptstück: Die besonderen Ursachen der einheimischen Cholera.

§ 13. Der Streit um den Vibrio Finklers und Priors . . . . . . . 24-26

§14. Andere Vibrionen . . . . . . . . . . . . . . . . . 26-29

$\S 15$. Der Bacillus paratyphi B . . . . . . . . . . . . . . . 29-31

§16. Cholera nostras als Nitritvergiftung. . . . . . . . . . . $31-34$

IV. Hauptstũck: Das Krankheitsbild der einheimischen Cholera.

§17. Vorboten und Anfall . . . . . . . . . . . . . . . . 34-36

§18. Verlauf . . . . . . . . . . . . . . . . . . . . $36-38$

§ 19. Nachkrankheiten . . . . . . . . . . . . . . . . . $38-39$

§20. Der Leichenbefund . . . . . . . . . . . . . . . . 39-41

v. Hauptstück: Die Diagnose der einheimischen Cholera.

$\S 21$. Klinische Diagnose . . . . . . . . . . . . . . . . $41-42$

§ 22. Akute Arsenikvergiftung. . . . . . . . . . . . . . . 42-45

§ 23. Vergiftung durch andere Mineralgifte . . . . . . . . . . 45-48

§ 24. Vergiftung durch Pflanzengifte . . . . . . . . . . . . 48-50

\$25. Vergiftung durch verdorbene Fleischspeisen. . . . . . . . 50-52

$\S 26$. Fischvergiftung . . . . . . . . . . . . . . . . . . 52-53

§ 27. Wechselfiebercholera. . . . . . . . . . . . . . . . 53-54

§ 28. Akute Bauchfellentzündung . . . . . . . . . . . . . 54-56 
G. Sticker: Abhandlungen aus der Seuchengeschichte u. Seuchenlehre. II. Band: Die Cholera. 3

§ 29. Akute Bauchfellzerreißung . . . . . . . . . . . . . $56-57$

§3. Innere Darmeinklemmung . . . . . . . . . . . . . . . . . $57-59$

§ 31. Akute Darmeinschiebung und Darmverseblingung . . . . . $59-61$

§ 32. Asiatische Cholera . . . . . . . . . . . . . . . . $61-63$

§33. Aetiologische Unterscheidung in Hamburg . . . . . . . $64-67$

§34. Aetiologische Unterscheidung in Nietleben . . . . . . . $67-70$

VI. Ila uptstück: Die Prognose der einheimischen Cholera.

§ 35. Leichte und schwere Epidemien . . . . . . . . . . . 70-71

VII. Hauptstück: Die Prophylaxe der einheimischen Cholera.

§3. Boden, Wasser. . . . . . . . . . . . . . . . . $71-72$

VIII. Hauptstück: Die Therapie des einheimischen Choleraanfalles.

§37. Kausale Behandlung . . . . . . . . . . . . . . . $72-74$

§ 38. Symptomatische Behandlung . . . . . . . . . . . . $74-75$

Zweiter Teil: Die Kindercholera.

IX. Hauptstück: Geschichte und Epidemiologie der Kindercholera.

§39. Kindercholera als besondere Krankheit. . . . . . . . . 76- 77

§ 40. Lntwicklung der Seuche in den Großstädten Englands . . . 77- 81

$\S 41$. Kindercholera in Amerika und Europa . . . . . . . . . 82-85

X. Hauptstück: Die Ursachen der Kindercholera.

§42. Zeit und Ort ihrer Ausbrüche . . . . . . . . . . . . 85- 88

§ 43. Einfluß der Ernährungsweise . . . . . . . . . . . . . . $88-91$

$\S 44$. Erregende Bakterien und Giftbildung . . . . . . . . . $91-93$

XI. Hauptstück: Das Krankheitsbild der Kindercholera.

$\S 45$. Verlauf der Krankheit . . . . . . . . . . . . . . 93-96

§ 46. Der Leichenbefund . . . . . . . . . . . . . . . 96-98

XII. Hauptstück: Prophylaxe und Therapie.

§ 47. Säuglingspflege . . . . . . . . . . . . . . . . . 98-100

Dritter Teil: Die indische Cbolera.

\section{Erste Abteilung: Die indische Cholera als Seuche.}

XIII. Hauptstück: Das Wesen der indischen Cholera.

§ 48. Tatsachen und Theorien. . . . . . . . . . . . . . 101-104

XIV. Hauptstück: Die Geschichte der indischen Cholera.

$\S 49$. Die indische Cholera im Altertum . . . . . . . . . . 104-106

$\S 50$. Die indische Cholera im 16., 17. und 18. Jahrbundert . . . 106-109

§51. Auswanderung der indischen Cholera . . . . . . . . . 109-110

§5. Die erste Pandemie . . . . . . . . . . . . . . . 110-114

§53. Europäische Versuche der Abwehr . . . . . . . . . . 114-120

§54. Die zweite, dritte und vierte Pandemie . . . . . . . . 120-122

$\S 55$. Die fünfte Pandemie . . . . . . . . . . . . . . . 122-126

§56. Gegenwärtige Choleragefahr für Europa . . . . . . . . 126-129

XV. Hauptstück: Die geographische Verbreitung der indischen Cholera.

§57. Vorderindien ihre Heimat . . . . . . . . . . . . . 129-130

§58. Weitere Ansiedlungen . . . . . . . . . . . . . . 131-132

§59. Cholerasichere Gegenden und Orte . . . . . . . . . . 132-133 
XVI. Hauptstück: Die Epidemiologie der indischen Cholera.

§ 60. Die Ursache ihrer Auswanderung im Jahre 1817. . . . . 133-135

$\S$ 61. Bodentheorie und Kontagionismus . . . . . . . . . . 135-137

\$ 62. Die Heimat der Wandercholera. .. . . . . . . . . . 137-139

$\S$ 63. Überwintern der Cholera . . . . . . . . . . . . . . . . 139-142

$\S$ 64. Aussaaten der Cholera durch den Menschenverkehr. . . . 142-143

$\S 65$. Wege der Cholera an Wasserläufen . . . . . . . . . 143-146

\& 66. Örtliche Disposition . . . . . . . . . . . . . . . 146-148

§ 67. Zeitliche Disposition . . . . . . . . . . . . . . . 148-150

$\S 68$. Entwickelung und Gang der Choleraverseuchung . . . . . 150-153

§ 69. Choleraausbruch und Ansteckungsketten . . . . . . . . 153-155

§ 70. Schwere und leichte Ausbrüche. . . . . . . . . . . 155-160

§ 71. Winterausbrüche . . . . . . . . . . . . . . . . . . 161-162

$\S$ 72. Einwanderer und Auswanderer aus Choleraorten . . . . . 162-166

\& 73. Cholerakeimträger. . . . . . . . . . . . . . . . 166-170

$\S$ 74. Das Gesetz der Choleraübertragung . . . . . . . . . 170-172

§ 75. Cholerahäuser, Choleraschiffe . . . . . . . . . . . 172-176

$\S$ 76. Ansteckung von Person zu Person . . . . . . . . . 176-180

§ 77. Wäsche und Kleider als Überträger . . . . . . . . . 180-183

\$ 78. Tiere als Choleraträger . . . . . . . . . . . . . . 183-186

$\S$ 79. Fliegen als Choleravorbreiter. . . . . . . . . . . . 186-188

\$ 80. Trinkwasser als Keimvermittler . . . . . . . . . . . 188-192

$\$$ 81. Trinkwasser und Choleragift . . . . . . . . . . . . 192-195

$\S$ 82. Nahrungsmittel als Choleraüberträger . . . . . . . . . 195-197

$\S 83 . \quad$ Abtritte . . . . . . . . . . . . . . . . . . . . $197-200$

XVII. Hauptstüch: Die Bakteriologie der indischen Cholera.

§ 84. Die Entdeckung des Choleraerregers . . . . . . . . . 200-203

§ 85. Die Morphologie des Kommabazillus . . . . . . . . . 203-205

§ 86. Seine Entwicklung . . . . . . . . . . . . . . . 205-206

§ 87. Sein Gedeihen im Boden . . . . . . . . . . . . . 206-207

$\$$ 88. Sein Gedeihen auf Nahrungsmitteln . . . . . . . . . 207-209

\$ 89. Seine Verschleppung durch Fliegen . . . . . . . . . 209-211

$\S 90$. Sein Verhalten im Wasser . . . . . . . . . . . . $211-213$

§ 91. Die Trinkwassertheorie . . . . . . . . . . . . . . . 213-216

§ 92. Die Lehre von der Flußverseuchung . . . . . . . . . 216-218

§ 93. Übertragung des Choleravibrio durch Kontakt . . . . . . 218-220

§ 94. Ausdauer des Vibrio in Faekalien . . . . . . . . . . 220-221

§ 95. Seine Ansprüche an Nährstoffe . . . . . . . . . . . 221-221

§ 96. Seine chemischen und physikalischen Ansprüche . . . . . 223-226

§ 97. Sein Verhalten gegen Desinfektionsmittel . . . . . . . 226-227

§ 98. Sein Wachstum auf künstlichen Nährböden . . . . . . 227-230

§ 99. Seine Anreicherung . . . . . . . . . . . . . . . 230-233

$\S 100$. Mutationen und Variationen . . . . . . . . . . . . 233-236

§ 101. Chemische Leistungen, Nitritbildung . . . . . . . . . $236-238$

$\S$ 102. Haemolyse, Phosphorescenz . . . . . . . . . . . . 238-240

§ 103. Die Bakterioskopie des Choleravibrio . . . . . . . . . 240-241

$\S 104$. Vibrionenträger, Vibrionenausscheider . . . . . . . . 241-246

$\S 105$. Die Ansiedlung des Choleravibrio im menschlichen Körper . 246-248

$\S 106$. Sein Gedeihen im Dünndarm . . . . . . . . . . . 248-250

$\S 107$. Choleraexotoxin . . . . . . . . . . . . . . . . 250-254 


\section{Znrhistorischen Biologie der Krankheitserreger Materialien, Studien und Abhandlungen} gemeinsam mit V. FOSSEL, T. v. GYÖRY, W. HIS herausgegeben von KARL SUDH0FF and GE0RG STICKER

1. Heft M. -.40

2. Heft N. 1.40

3. Heft M. 2.-

4. Heft I. 1.-

5. Heft M. 2.50

6. Heft M. 1.40

Karl Sudhoff: Historik und Seuchenforschung Georg Sticker: Parasitologie und Loimologie

Georg Sticker: Die Bedeutung der Geschichte der Epidemien für die heutige Epidemiologie

Grafton Elliot Smith und Mare Armand Ruffer:

Pott'sche Krankheit an einer ägyptischen Mumie. Vorausgesehiekt: Karl Sudhoff: Zur Einführung und Orientierung

Georg Sticker: Zur historischen Biologie des Erregers der pandemischen Influenza

Karl Sudhoff: Mal Franzoso in Italien in der ersten Hälfte des 15. Jahrhunderts. Ein Blatt aus der Geschichte der Syphilis.

Tiberius von Györy: Der Morbus Brunogallicus (15\%7). Ein Beitrag zur Geschichte der Syphilisepidemien.

\section{Gesundheit und Erziehung}

2. Auflage.

Eine Vorschule der Ehe

Geb. 5 M.

\section{von Georg Sticker Bonn}

In dem gediegen ausgestatteten Buche lernen wir den bekannten, vielseitigen Kliniker wieder von einer neuen Seite kennen und schätzen, nämlich als einen Popularphilosoph der Medizin oder besser Popularhygieniker, der von Herz zu Herz zu reden versteht. Es ist nicht leicht, für den gebildeten Mann populär zu schreiben. Hier ist tiefes Denken, überzeugende Kraft der Ausführungen mit edler Sprache und Originalität der Form verknüpft. Stickers Buch zu lesen ist ein Genuß, den wir allen Kollegen von Herzen gönnen. Prof. Dr. J. Pagel (Berlin) in der Deutschen Ärzte-Zeitung.

\section{Über Naturheilkunst}

Geh. 3 M.

Geb. 4 M.

Ungewöhnliche Begabung für den richtigen populären Ton, ein historisch und allgemein literarisch sehr reiches Wissen, aus dem die für die Veranschaulichung dienlichen Beispiele, man möchte sagen, aus dem Ärmel geschüttelt erscheinen, ein glänzender Stil, das sind die Vorzüge des trefflichen Büchleins. Münch. Medizin. Wochenschrift. 



\section{Abhandlungen}

aus der

\section{Seuchengeschichte und Seuchenlehre}

von

Georg Sticker

\section{Band: Die Cholera}

Yit 4 Textbildern

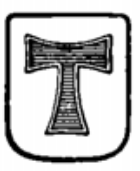

Gießen 1912

Verlag von Alfred Töpelmann (vormals J. Ricker) 
Buchdruckerei des Waisenhauses in Halle a. d. S. 